\title{
The Difference between Hot and Cold Deformation and its Effect on Microstructure and Mechanical Properties of Near Beta Titanium Alloy
}

\author{
A.S.Grebenchshikov ${ }^{\mathrm{a}, *}$, M.O.Leder ${ }^{\mathrm{a}}$, A.V.Volkov ${ }^{\mathrm{a}}$ \\ ${ }^{\text {a }}$ PSC VSMPO-AVISMA Corporation \\ *'grebenshchikov_as@vsmpo.ru
}

\begin{abstract}
$\underline{\text { Abstract }}$
An investigation of cold and hot deformation and the subsequent heat treatment including the annealing at the $(\alpha+\beta)$ - field temperature and ageing of VST55531 alloy showed that the material after the cold drawing without the heat treatment has the extremely low values of uniform elongation and during the tensile test the strain becomes localized at a neck upon $0.2 \%$ strain. The ageing of coldworked material improves the strength and increases a limit of uniform deformation and generally has a beneficial effect on a strengthto-ductility ratio of alloy probably due to achieving a special morphology of secondary $\alpha$-phase. The obtained values of the combination of strength and ductility in the cold-worked material at 50\% strain reduction degree are $1625 \mathrm{MPa}$ for UTS, 8\% - El, 36\% - RA, that exceed significantly the values obtained for the hot-worked and as-annealed material, and are attractive for application in products that require the increased strength and ductile characteristics.
\end{abstract}

\section{Introduction}

Due to capability of achieving the high strength and ductile characteristics the near $\beta$ - titanium alloys are advanced materials for manufacturing of wide range of products beginning with large-sized die forgings and ending with miniature fasteners [1]. To produce the semi-finished products of titanium alloys it is necessary to fabricate a required shape for an ingot and create the structure in the material that will provide the desired set of properties. A variety of ways for deformation effect on the material viz. a variation of temperature, rate and degree of deformation as well as the heat treatment conditions namely the variation of temperature, soaking time, heating \& cooling rate, determine the wide range of both the structural condition and obtained mechanical properties. The hot deformation ( $\left.\sim 0.5 \mathrm{~T}_{\text {melt }}\right)$ is a main method for manufacturing of stocks of titanium alloys, and mechanisms of its effect on the structure formation are sufficiently studied. Thus, for example, as for Ti-55531 alloy to study the influence of temperature - rate conditions the work for elaboration of processing map for deformation within the temperature range of $\beta$ - $\& \alpha+\beta$ - fields was conducted [2]-[4]. The cold deformation of $\beta$ - titanium alloys in its turn is used for manufacturing of wire, springs and seamless tubes and allows to expedite the ageing process for alloys high in $\beta$ - stabilizers [1]. A typical picture for ageing of cold-worked $\beta$ - titanium alloy is a growth of strength and a fall of ductility because of precipitation hardening process [5], [6].

The present investigation is aimed at research of microstructure evolution after the hot and cold deformation as well as upon the annealing and ageing of VST55531 alloy and its effect on the mechanical properties under tension at room temperature. 


\section{$\underline{\text { 2. Experimental procedure }}$}

The alloy VST55531, having a chemical composition of (in weight \%) 5.3Al, 4.8Mo, 5.0V, 2.9 Cr, $1 \mathrm{Zr}, 0.3 \mathrm{Fe}, 0.07 \mathrm{O}$, $0.004 \mathrm{~N}, 0.007 \mathrm{C}$, remainder Ti, was produced by a vacuum-arc remelt method. The melted ingot was deformed at the temperatures of $\beta$ $\&(\alpha+\beta)$ field, the final deformation of ingot was made to manufacture forged billets for rolling. A Ø22 mm bar was produced from the machined billets by rolling operations. This bar had the temperature of deformation completion in the $(\alpha+\beta)$ field, and the structure of which is shown in Fig. 1a. Then the bar underwent the hot rolling in the $(\alpha+\beta)$ - field to $\varnothing 12 \mathrm{~mm}$ at $70 \%$ degree of reduction. The stock for cold-worked material was the $\varnothing 12.8 \mathrm{~mm}$ bar produced by rolling at the $\beta$ - field temperatures with the subsequent annealing in the $(\alpha+\beta)$ field; the structure of which is presented in Fig. 1b. Subsequently the bar was subjected to the cold drawing at the $30 \% \& 50 \%$ strain degree (hereinafter referred to as a low cold work - LCW and heavy cold work - HCW respectively). For the investigation the initial states after the hot and cold work and also after the subsequent annealing and aging at the $(\alpha+\beta)$ - field temperatures were selected. The tensile test was carried out at the room temperature on cylindrical specimenswith4 $\mathrm{mm}$ diameter of working part as per the procedure of ASTM E8/E8M. The microstructure examination was performed using Carl Zeiss Sigma 300VP scanning - electron microscope (SEM) equipped with Hikari Pro attachment for EBSD analysis. Images were made in a contrast of back scattered electrons (BSE) at the accelerating voltage of $5 \mathrm{kV}$. The EBSD maps were acquired at the accelerating voltage of $20 \mathrm{kV}$ and beam current of 10 $\mathrm{nA}$, step size of $0.2 \mathrm{~mm}$. The maps were subjected to cleaning from noise points by way of Clean Up - Grain CI Standardization. The specimens for EBSD analysis underwent electrolytic polishing in the $\mathrm{HClO}_{4}+\mathrm{CH}_{3} \mathrm{COOH}$ solution at the voltage of $60 \mathrm{~V}$ and the temperature of $0^{\circ} \mathrm{C}$. A calculation of secondary phase particles density was performed in the Olympus Stream Motion program. A fraction of recrystallized structure was determined by the Grain Orientation Spread (GOS) maps. The value of GOS $<2^{\circ}$ at the critical grain-boundary angle (GSTA) $7^{\circ}$ was taken as a criterion for recrystallized grain.

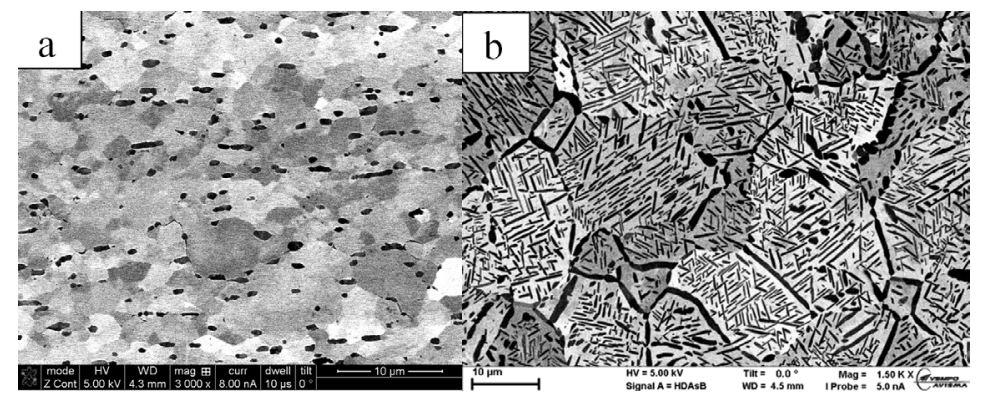

Figure. 1 SEM BSE images of undeformed specimens a) before hot rolling b) before cold drawing

\section{$\underline{\text { 3. Results }}$}

A fraction of primary alpha phase in the initial material before drawing was $37 \%$. The morphology of primary alpha phase is lamellar (Fig. 1b) with the presence of individual globules. The coarsest lamellas are observed along the boundaries of $\beta$ - grains, an average size 
of which is $15 \mathrm{~mm}$. The drawing leads to an extension of primary $\alpha$-phase lamellas as well of $\beta$-grains in the direction of drawing. The $\alpha$ - phase lamellas located perpendicular to the deformation direction assume a corrugated shape, and at the interfacial boundary the typical steps occur that indicate exit points of slip bands (Fig.2c). In the material after ageing along the slip bands the elongated precipitations of secondary $\alpha$ - phase are observed which form typical banding (Fig.2b see arrows). A secondary alpha phase has an atypical morphology, in which there are no pronounced colonies of the secondary $\alpha$ - phase, while the particles enframe areas resembling cells, that have sizes about 20-30 $\mathrm{nm}$. Most probably the heating to the ageing temperature leads to an activation of recovery processes, an initial stage of which is a formation of cellular dislocation structure [7]. The boundaries of cells have the increased density of dislocations and as a consequence are preferred places for origin of secondary $\alpha$ - phase particles.

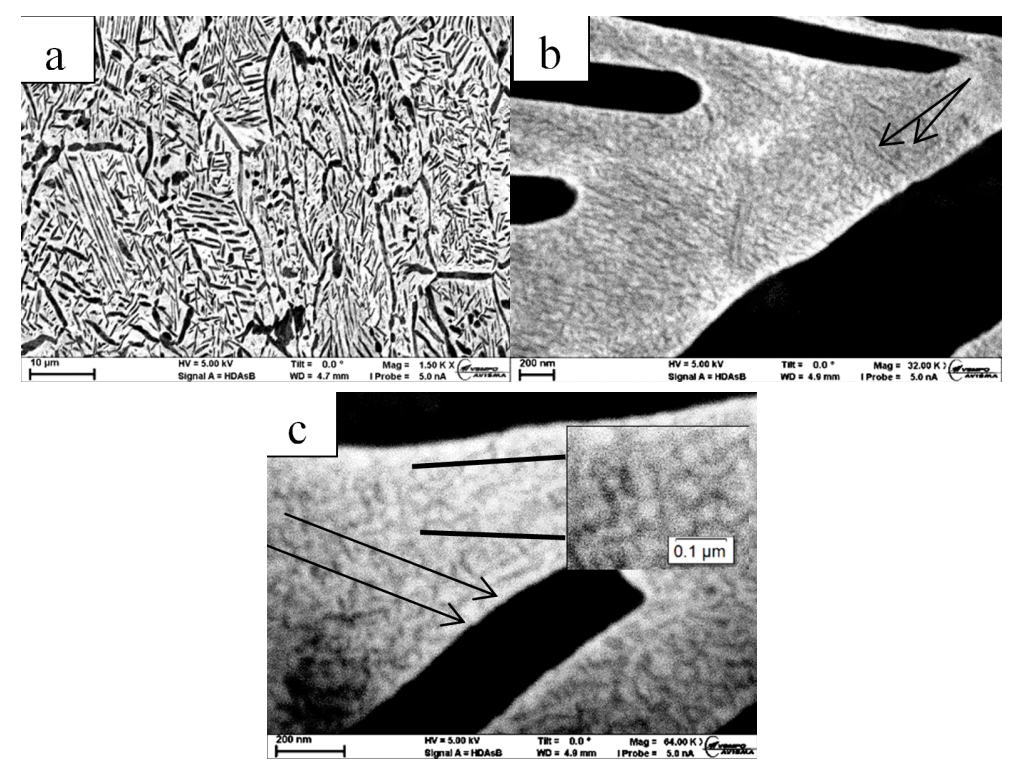






Figure 2 SEM BSE images showing microstructure of a,b,c - cold deformed $30 \%$ reduction+aged, d,e,f - cold deformed $50 \%$ reduction + aged.

The increase of cold work reduction to $50 \%$ leads to the further extension of $\beta$-grains and $\alpha$ - lamellas in the drawing direction (Fig. 2d). The steps at the boundaries of primary $\alpha$ - phase lamellas become more pronounced (Fig.2e). Also, there are areas in the microstructure where the abrupt lamellas of the $\alpha$-phase can be observed that may indicate the localized shear bands (Figure $2 \mathrm{~d}$, see arrows). The presence of such areas gives evidence of strain nonuniformity in the drawing process. The products of ageing have a similar morphology in comparison with the LCW material (Fig. 2f). In view of complexity for calculation of geometrical sizes of particles due to a resolution limitation of scanning-electron microscope, the number of particles per unit surface area of metastable $\beta$ phase was calculated. In the approximation that an increase of dispersion of precipitates results in the decrease of its linear sizes and inter-particle distance the density of particles may serve as a criterion for comparison of dispersion of secondary $\alpha$-phase in the different conditions of material [8]. Based on results of calculation of particles density the increase of cold work reduction does not result in the increase of dispersion of secondary $\alpha$ - phase at the same ageing temperature (Fig. 4a). Most probably such picture is a consequence of nonuniformity of cold work in the HCW material. Due to the strain localization the density of dislocations in the $\beta$ - phase and as a consequence the number of secondary alpha phase nucleation places do not increase in comparison with the LCW material.

After annealing and ageing a significant change of secondary $\alpha$-phase morphology is observed. The two key types differing in dispersion can be observed - the secondary $\alpha$ - phase lamellas in the recrystallized grains are more thick and extended in comparison with the ones in the polygonized grains (Fig.3). This picture is typical both for the hot worked and cold worked material. It should be noted that along the low-angle boundaries the density of secondary $\alpha$-phase particles is $\sim 3$ times as much than in a body of polygonized subgrain and comparable with the density of particles in the aged cold worked material. At that the density of particles in the recrystallized grains is about 2 times lower than in the polygonized ones (Fig. 4a). Apart from the increased density, the particles along the boundary have also a similar orientation and grow perpendicular to the boundary line (Fig. 3c). Generally, the density of particles in the material after the annealing and ageing is similar within the type of $\beta$ - phase structure irrespective of deformation history. At the 
same time, the fraction of particular type of structure differs after the annealing. Thus the fraction of recrystallized grains after the annealing of cold worked material after $30 \% \& 50 \%$ reduction differs 2.5 times (Fig. 4b). The increase of recrystallized grains fraction in HCW material after annealing can be explained by increased driving force of recrystallization due to high work hardening.[7]. The fraction of recrystallized grains in the material after the hot rolling is about $12 \%$ that is due to dynamic recrystallization during heating and deformation. In whole, the fraction of recrystallized grains is similar in HCW material and hot rolled material after the annealing. In addition to the recrystallization process during the annealing operation the recovery process also takes place, accompanied by the formation of subgrains surrounded by the low-angle boundaries (Fig. 6). The observed fall of density of low-angle boundaries at the annealing of cold worked material (Fig. 5a) is related to the growth of subgrains at the high temperature annealing, the average size of which increases from $1.5 \mathrm{~mm}$ in the LCW condition to $6.5 \mathrm{~mm}$ in the LCW + annealed condition. The annealing of cold worked material is accompanied by the drop in local misorientation (Kernel average misorientation (KAM)) (Fig. 5b). It is interesting that a behavior of KAM parameter at the annealing is extremely close for the LCW and HCW material at a different kinetics of recovery and recrystallization. It is apparently related to that the KAM parameter correlates with the density of geometrically necessary dislocations [9] not perceiving at that the density of statistically stored dislocations (SSDs). The SSDs are an important component of work hardening, the evaluation of which is outside the scope of the present investigation.
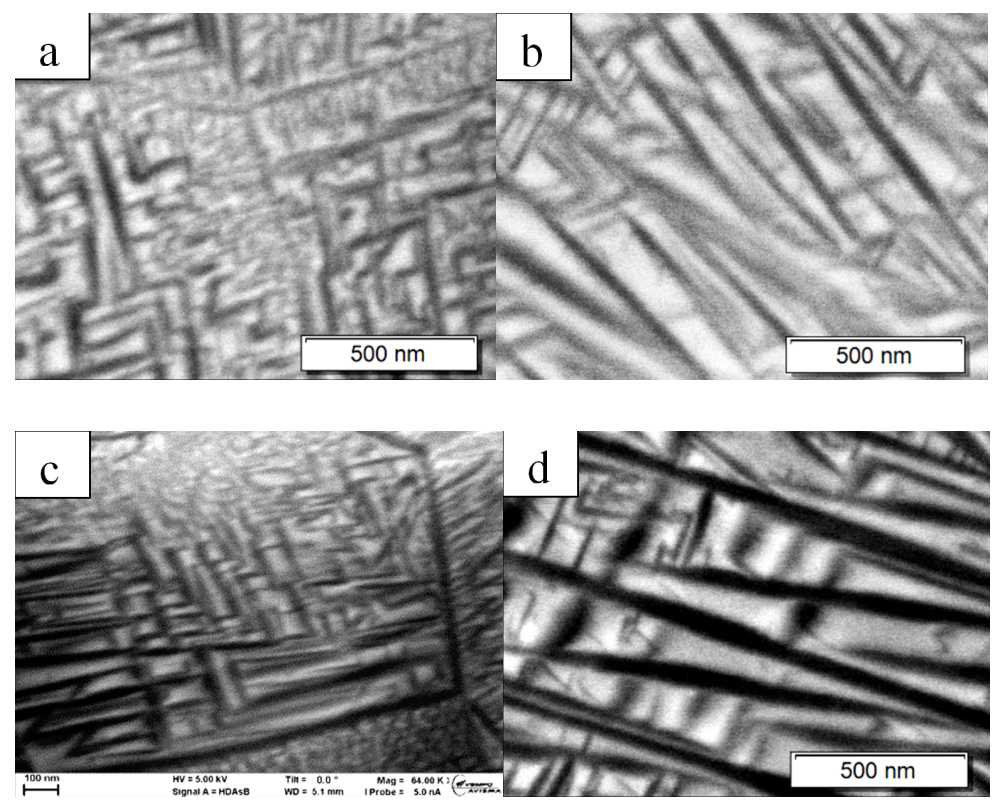

Figure 3 SEM BSE images showing microstructure of hot rolled (a,b) $70 \%$ reduction and cold drawn $50 \%$ reduction (c, d) after anneal + ageing. (a,c) - recovered grains, (b,d) - recrystallized grains 


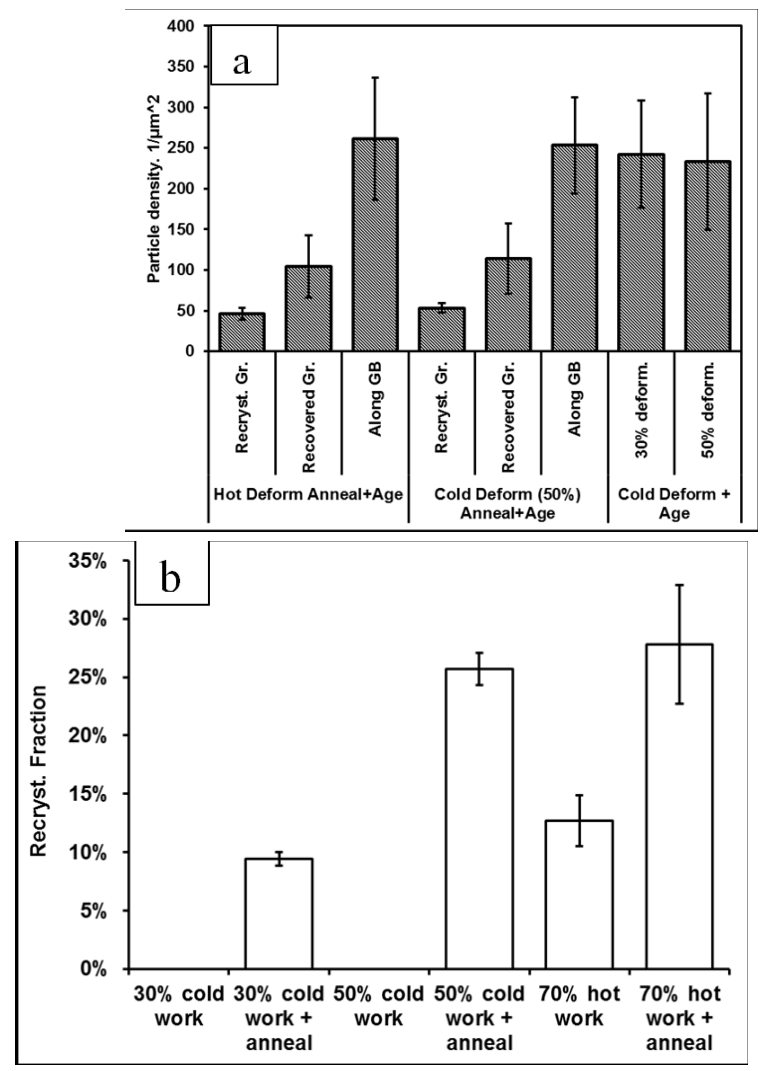

Figure 4 a - secondary $\alpha$ - phase precipitation density in different regions of microstructure $b$ - recrystallized grains fraction in initial state and after annealing 



Figure 5 a - boundary density values for different material conditions b-KAM values for different material conditions

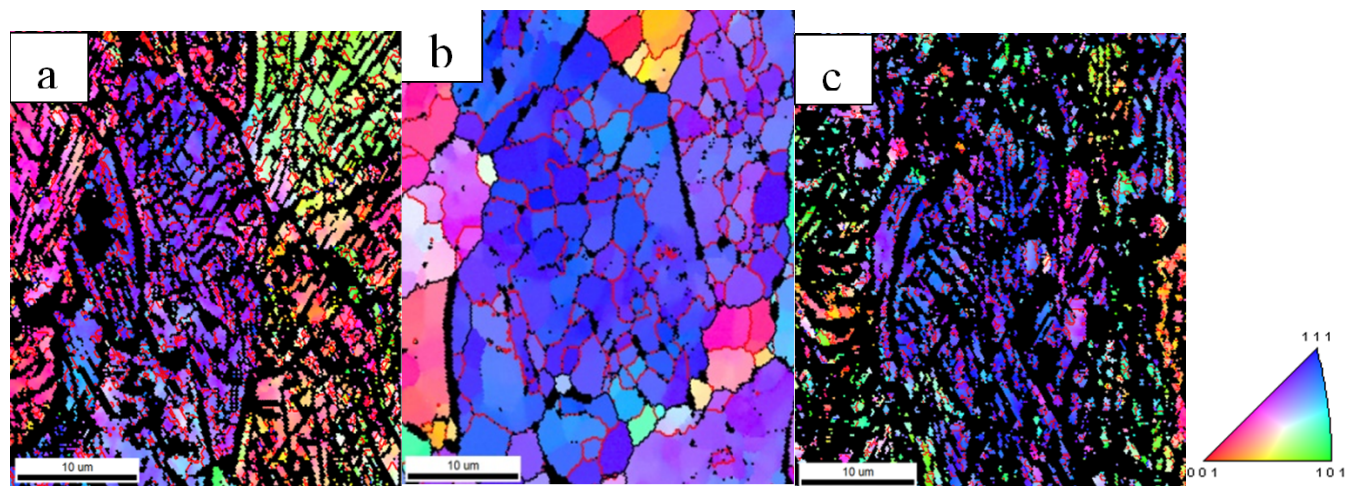






Figure 6 b,c - IPF map with grain boundaries showing LAB (shown in yellow), HAB (shown inblue) evolution with annealing (a - LCW, b -

LCW + anneal, $\mathrm{c}-\mathrm{HCW}, \mathrm{d}-\mathrm{HCW}+$ anneal, $\mathrm{e}-$ hot rolled, $\mathrm{f}-$ hot rolled+anneal)

A distinctive feature of mechanical characteristics of cold-worked material is an early transition to the localized strain region; the uniform elongation is $0.2-0.3 \%$ (Fig. $7 b$ ). One may assume that at the tensile test the early localization of flow happens in the localized shear bands found in the structure of cold worked material. Because of the heating to the ageing temperature the dislocation structure of material changes and the uniform elongation at testing increases to $1.7-1.9 \%$. The total elongation remains similar to the non-aged specimen regardless of strength growth by $200 \mathrm{MPa}$. Also the increased ductility of aged cold-worked material as compared to the annealed one is noteworthy (Fig. 7b). And also this picture is typical both for the LCW material and the HCW one. The special morphology of secondary $\alpha$ - phase in the cold-worked material probably has a beneficial effect on the alloy ductility at the roomtemperature tensile.
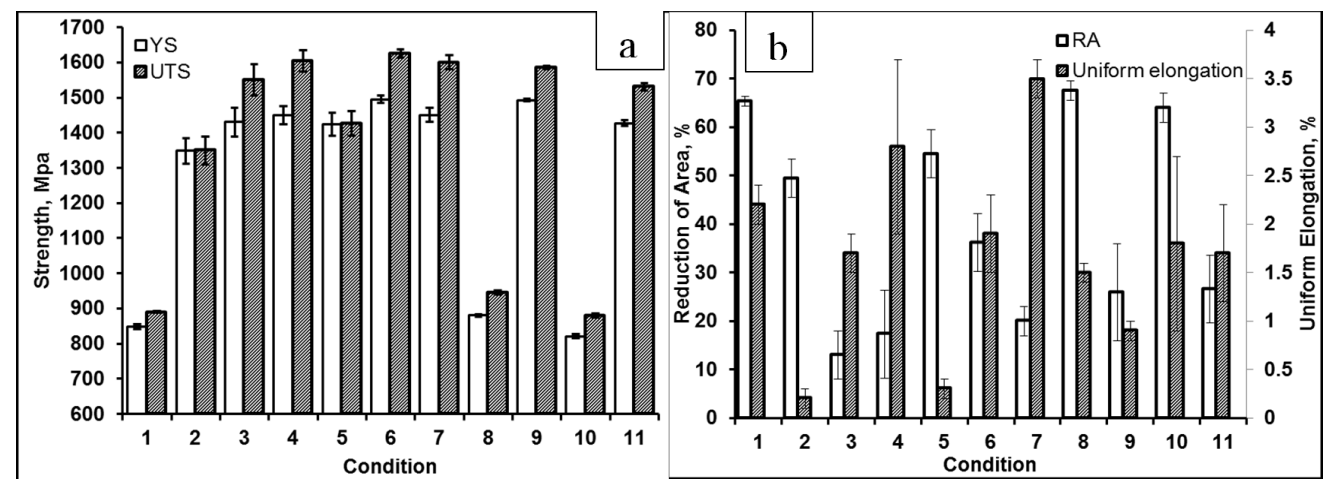

Figure 7 Strength (a) and ductility (b) values for different material conditions: 1- Initial condition, 2 - Cold D. $30 \%$ red., 3 - Cold D $30 \%$ red. + ageing, 4 - Cold D. 30\% red. + anneal + ageing 5 - Cold. D. 50\% red. 6 - Cold D 50\% red. + ageing 7 - Cold D. 50\% red. + anneal + ageing, 8 Hot D. 70\% red. , 9 - Hot D. 70\% red. + ageing, 10 - Hot. D. 70\% red. + anneal , 11 -Hot D. 70 Hot. D. $70 \%$ red. + anneal + ageing

\section{Discussion}


The morphology and sizes of structural constituents are a factor that has a significant impact on the mechanical properties of metal materials. Particularly the dispersion of secondary $\alpha$ - phase precipitating at the ageing of near- $\beta$ titanium alloys has a determining influence on its strength. What do the dispersion of secondary $\alpha$-phase depend on? In the first place, it depends on the temperature of ageing. The effect of reduced temperature of ageing is a low diffusion mobility of alloying elements that in its turn leads to the formation of more number of nucleation sites for secondary $\alpha$-phase particles. However the results of present investigation and also in [6], [10] show that the secondary $\alpha$-phase dispersion is determined also by the dislocation structure of $\beta$ - matrix. In the annealed material the recrystallized grains, having the reduced density of dislocations, generally have the reduced dispersion of secondary $\alpha$-phase, i.e. the density of particles in them is more than 2 times lower as compared to the recovered structure. And also the availability of low-angle boundaries has a significant impact, viz. the secondary $\alpha$ - phase precipitating along the boundary has a similar orientation, at that the density of particles along the boundaries is 6 times higher than in the recrystallized $\beta$ - matrix. Consequently, the availability of low-angle boundaries shall cause the difference in the strength characteristics of aged material. The data obtained in the present study show that regardless of twofold higher density of low-angle boundaries (LAB) in the cold deformed material after annealing compared to the hot rolled material the strength is higher merely by $4.5 \%$. At that comparison of the cold worked material after $30 \%$ \& $50 \%$ reduction shows that with the different fraction of recrystallized grains and as a consequence the different volume fraction of grains with more coarse secondary $\alpha$-phase, the strength after the annealing and ageing in the those states is practically identical.

The strength of cold worked material after the ageing is higher than after the ageing of annealed material considering the different volume fraction of primary $\alpha$-phase. At the same fraction of primary $\alpha$-phase the strength in a general way will be higher at the more strong $\beta$ - transformed matrix [11]. The difference in the fraction of primary $\alpha$-phase between the aged cold deformed material and the annealed one is about $30 \%$, at that the strength after the ageing is nearly similar. In view of the fact that the work hardening of primary $\alpha$-phase makes also an effect on the total hardening, the precipitation age hardening of metastable $\beta$ - matrix makes the largest contribution. The density of particles in the aged cold worked material is similar irrespective of the strain reduction and higher $2-5$ times than in the material after the annealing and ageing that causes the increased strength of these states. The strength increase at the ageing of cold deformed material is about $200 \mathrm{MPa}$, while the strength increase due to the ageing of hot rolled material is about $600 \mathrm{MPa}$ at the same temperature of ageing. The lower ageing strengthening of cold rolled material is explained by the competition of softening processes of recovery and hardening by the dispersion hardening.

Not only the strength level but also the ductility is important in terms of applicability of material in the actual products. The present paper gives evidence that the cold worked material after the ageing possesses the increased ductility at the same strength level compared to the annealed material after the ageing. Moreover, the increase of cold work reduction degree leads to yet more increase of ductility. This effect disappears after the annealing and is absent in the hot worked material. By all appearances, the specific morphology of secondary $\alpha$-phase, formed at the ageing, has a beneficial effect on the ductility. However, understanding of mechanism for ductility increase requires further research.

\section{$\underline{\text { 5. Conclusion }}$}

The investigation conducted in the present paper shows that the cold deformation and ageing allows to obtain the increased values of ductility at the same strength compared to the hot worked material that most probably relates to the formation of dislocation cell structure of $\beta$ - phase with subsequent precipitation of secondary $\alpha$-phase along the cell boundaries. However, it should be taken into account that an instability of deformation at the cold drawing that manifests itself in the deformation localization shall lead to the 
inhomogeneity of structure and finally to the nonuniformity of mechanical properties. A set of testing statistics is required to confirm this assumption.

The annealing of cold worked material leads to the similar structure and mechanical properties as compared to the hot rolled material and results in a loss of benefit of cold worked material in terms of mechanical properties.

\section{References}

[1] J. D. Cotton et al., "State of the Art in Beta Titanium Alloys for Airframe Applications," JOM, vol. 67, no. 6, pp. 1281-1303, Jun. 2015.

[2] M. Dikovits, C. Poletti, and F. Warchomicka, "Deformation Mechanisms in the Near- $\beta$ Titanium Alloy Ti-55531," Metall. Mater. Trans. A, vol. 45, no. 3, pp. 1586-1596, Mar. 2014.

[3] Y. Li, X. Ou, S. Ni, and M. Song, "Deformation behaviors of a hot rolled near- $\beta$ Ti-5Al-5Mo-5V-1Cr-1Fe alloy," Mater. Sci. Eng. $A$, vol. 742, pp. 390-399, Jan. 2019.

[4] F. Warchomicka, C. Poletti, and M. Stockinger, "Investigation of the hot deformation behaviour in Ti-5Al-5Mo-5V-3Cr-1Zr," Mater. Sci. Eng. A, vol. 528, no. 28, pp. 8277-8285, Oct. 2011.

[5] Z. Y. Song, Q. Y. Sun, L. Xiao, L. Liu, and J. Sun, "Effect of prestrain and aging treatment on microstructures and tensile properties of Ti-10Mo-8V-1Fe-3.5Al alloy," Mater. Sci. Eng. A, vol. 527, no. 3, pp. 691-698, Jan. 2010.

[6] T. W. Xu, J. S. Li, S. S. Zhang, F. S. Zhang, and X. H. Liu, "Cold deformation behavior of the Ti-15Mo-3Al-2.7Nb-0.2Si alloy and its effect on $\alpha$ precipitation and tensile properties in aging treatment," J. Alloys Compd., vol. 682, pp. 404-411, Oct. 2016.

[7] F. J. Humphreys and M. Hatherly, Recrystallization and related annealing phenomena, 1st ed. Oxford, OX, UK; Tarrytown, N.Y., U.S.A: Pergamon, 1995.

[8] S. A. Mantri et al., "Tuning the scale of $\alpha$ precipitates in $\beta$-titanium alloys for achieving high strength," Scr. Mater., vol. 154, pp. 139-144, Sep. 2018.

[9] T. J. Ruggles and D. T. Fullwood, "Estimations of bulk geometrically necessary dislocation density using high resolution EBSD," Ultramicroscopy, vol. 133, pp. 8-15, Oct. 2013.

[10] W. Yu, M. Q. Li, and J. Luo, "Effect of deformation parameters on the precipitation mechanism of secondary $\alpha$ phase under high temperature isothermal compression of Ti-6Al-4V alloy,” Mater. Sci. Eng. A, vol. 527, no. 16-17, pp. 4210-4217, Jun. 2010.

[11] J. Gurland, "A structural approach to the yield strength of two-phase alloys with coarse microstructures," Mater. Sci. Eng., vol. 40, no. 1, pp. 59-71, Sep. 1979. 